Case Report

\title{
Pulmonary Phaeohyphomycosis Caused by Phaeoacremonium in a Kidney Transplant Recipient: Successful Treatment with Posaconazole
}

\author{
Saivaralaxmi Monaganti, ${ }^{1}$ Carlos A. Q. Santos, ${ }^{1}$ Andrea Markwardt, ${ }^{2}$ \\ Morgan A. Pence, ${ }^{3}$ and Daniel C. Brennan ${ }^{1}$ \\ ${ }^{1}$ The Department of Medicine, Washington University School of Medicine, 660 S. Euclid Avenue, \\ Renal Division/Campus Box 8126, St. Louis, MO 63110, USA \\ ${ }^{2}$ Barnes-Jewish Hospital, Center for Outpatient Health, 4901 Forest Park Avenue, 5th Floor, St. Louis, MO 63108, USA \\ ${ }^{3}$ Department of Pathology \& Immunology, Washington University School of Medicine, 660 S. Euclid Avenue, \\ Campus Box 8118, St. Louis, MO 63110, USA
}

Correspondence should be addressed to Daniel C. Brennan; dbrennan@dom.wustl.edu

Received 18 December 2013; Revised 3 March 2014; Accepted 21 March 2014; Published 14 May 2014

Academic Editor: Samy S. Iskandar

Copyright (C) 2014 Saivaralaxmi Monaganti et al. This is an open access article distributed under the Creative Commons Attribution License, which permits unrestricted use, distribution, and reproduction in any medium, provided the original work is properly cited.

\begin{abstract}
We report a rare case of pulmonary phaeohyphomycosis in a 49 -year-old woman 6 years after kidney transplantation. She presented with dyspnea, cough, and fatigue. Her chest CT scan revealed nodular opacities in the right upper lung. A fine needle aspirate biopsy culture yielded Phaeoacremonium and surgical pathology of the biopsy showed chronic inflammation. We successfully treated her with posaconazole and managed drug interactions between posaconazole and tacrolimus. This is the second reported case of biopsyproven pulmonary infection by Phaeoacremonium in a kidney transplant recipient and successfully treated with posaconazole.
\end{abstract}

\section{Background}

Phaeoacremonium species are well known plant pathogens causing stunted growth and dieback of various woody hosts especially grapevines and have been isolated from necrotic woody tissue of Prunus species $[1,2]$. Phaeoacremonium species are dematiaceous fungi characterized by the presence of melanin or melanin-like pigments and are widely distributed in the environment particularly in soil, wood, and decomposing plant debris. Phaeohyphomycosis is a collective term for cutaneous, subcutaneous, and systemic disease caused by dematiaceous fungi. Pulmonary phaeohyphomycosis is a rare opportunistic infection of immunocompromised hosts. A review of 34 cases of dematiaceous fungal infections in organ transplant recipients revealed an overall mortality of $57 \%$ among patients with systemic disease and $7 \%$ among those with skin, soft-tissue, or joint infections [3].

This is only the second case of biopsy-proven pulmonary infection by Phaeoacremonium in a kidney transplant recipient and the first report of successful treatment with posaconazole. Moreover, management of drug interactions between posaconazole and tacrolimus was successfully done, thereby preventing supratherapeutic levels of tacrolimus and avoiding kidney injury.

\section{Case Report}

A 49-year-old Caucasian female who underwent a living related kidney transplant 6 years before presented with progressive dyspnea, cough, and fatigue over 6 months that failed to improve after the administration of several antibiotic courses. She had been on tacrolimus and prednisone for maintenance immunosuppression. She lived in a rural area, had exposure to chicken sheds and barns, and was a gardener. A chest CT scan revealed nodular opacities in the right upper lobe (Figure 1(a)), and she underwent bronchoscopy with bronchoalveolar lavage and transbronchial fine needle aspiration biopsy of the right upper lobe nodules.

Phaeoacremonium species grew from the biopsy culture within four days of incubation. Identification was assigned 


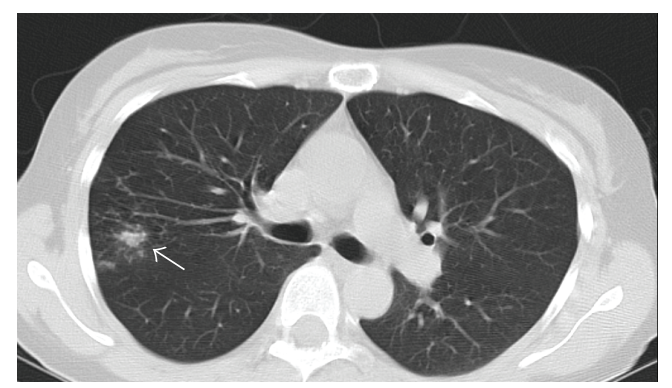

(a)

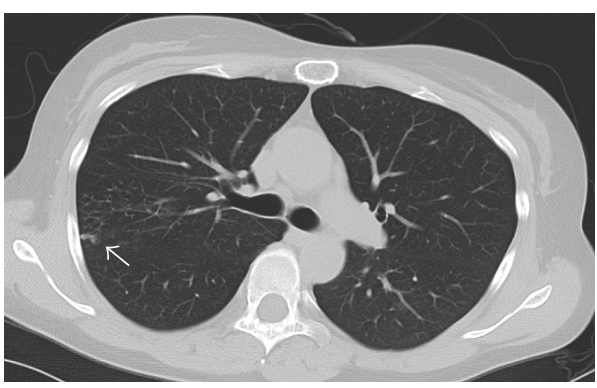

(b)

FIGURE 1: (a) Chest CT scan before starting posaconazole showing reticulonodular opacities in the right upper lobe. (b) CT scan one month after starting posaconazole showing resolution of most of the opacities.

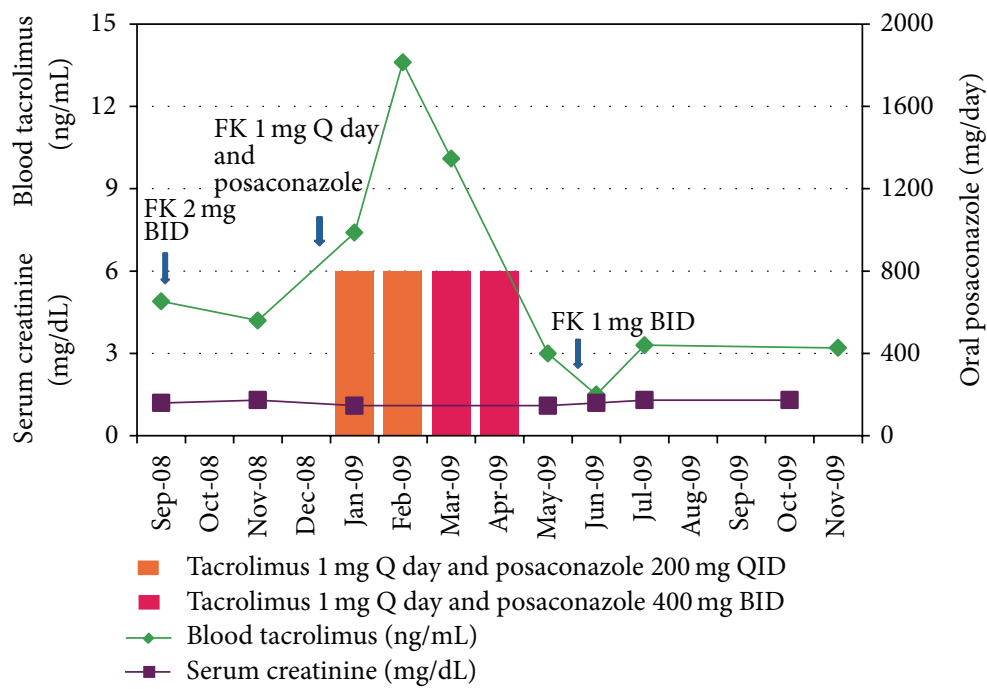

FIGURE 2: Graph showing serum creatinine and blood tacrolimus levels before, during, and after posaconazole treatment with tacrolimus dose adjustments.

based on macroscopic and microscopic morphology. Initially, the surface of the mold was olive in color, becoming greyishblack upon subculture. The texture was velvety, and the reverse was black. Microscopically, pigmented hyphae with tapering, funnel-shaped phialides were observed, and conidia were hyaline and oblong, forming clusters at the tip of the phialides. Macroscopic and microscopic morphology was consistent with Phaeoacremonium species. Surgical pathology of the biopsy showed chronic inflammation but no fungal hyphae. Culture for acid fast bacilli from the biopsy specimen was negative for mycobacteria. Culture of bronchial fluid yielded Dactylaria constricta and few Mycobacterium aviumintracellulare complex.

Given that her biopsy culture yielded Phaeoacremonium and showed chronic inflammation, we started oral posaconazole $200 \mathrm{mg}$ QID and reduced her tacrolimus dose from $2 \mathrm{mg}$ BID to $1 \mathrm{mg} Q$ day. A repeat chest CT scan one month after the institution of antifungal therapy showed improvement (Figure 1(b)), and she reported reduced cough and shortness of breath. Two months after commencing treatment, we changed her posaconazole dose to $400 \mathrm{mg}$ BID for greater ease of administration. She received posaconazole for 4 months and her symptoms resolved (Figure 2).

\section{Discussion}

To et al. reported the first case of biopsy-proven Phaeoacremonium parasiticum lung infection in a kidney transplant recipient. In contrast to our case, the patient was severely immunocompromised due to chemotherapy for posttransplant lymphoproliferative disease. He showed initial improvement with voriconazole and caspofungin but succumbed after a prolonged period of neutropenic fever. Shah et al. described a case of probable Phaeoacremonium lung infection in a lung transplant recipient. The patient developed cavitary lung nodules in the native lung a few months after single lung transplantation. Biopsy of one of the nodules showed chronic inflammation with possible granulomatous lesions. Phaeoacremonium parasiticum grew only from the bronchoalveolar lavage culture but not from the biopsy culture. The patient improved after the administration of voriconazole and caspofungin $[21,26]$. 
TABLE 1: Skin and subcutaneous infection, osteomyelitis, endophthalmitis, and onychomycosis due to Phaeoacremonium species.

\begin{tabular}{|c|c|c|c|c|c|c|c|}
\hline Number & Reference & Species & Age/sex & $\begin{array}{l}\text { Underlying } \\
\text { condition }\end{array}$ & Clinical disease & Treatment & Outcome \\
\hline 1 & $\begin{array}{l}\text { Padhye et al., } \\
1998 \text { [4] }\end{array}$ & P. inflatipes & $83 / \mathrm{F}$ & None & $\begin{array}{l}\text { Subcutaneous infection } \\
\text { of the foot }\end{array}$ & Excision & $\begin{array}{l}\text { Complete } \\
\text { healing }\end{array}$ \\
\hline 2 & $\begin{array}{l}\text { Matsui et al., } \\
1999[5]\end{array}$ & P. rubrigenum & $61 / \mathrm{F}$ & $\begin{array}{l}\text { Rheumatoid } \\
\text { arthritis }\end{array}$ & $\begin{array}{l}\text { Subcutaneous mass of } \\
\text { the foot }\end{array}$ & $\begin{array}{l}\text { Excision } \\
\text { Itraconazole } \\
\text { Fluconazole }\end{array}$ & Recurred \\
\hline 3 & $\begin{array}{l}\text { Kitamura et al., } \\
2000[6]\end{array}$ & P. parasiticum & $59 / \mathrm{F}$ & None & $\begin{array}{l}\text { Subcutaneous nodule } \\
\text { below the knee }\end{array}$ & Excision & $\begin{array}{l}\text { Complete } \\
\text { healing }\end{array}$ \\
\hline 4 & $\begin{array}{l}\text { Guarro et al., } \\
2003 \text { [7] }\end{array}$ & P. aleophilum & $19 / \mathrm{M}$ & None & $\begin{array}{l}\text { Fistulized nodule on the } \\
\text { ankle }\end{array}$ & $\begin{array}{l}\text { Excision (six } \\
\text { times) } \\
\text { Itraconazole }\end{array}$ & Cured \\
\hline 5 & $\begin{array}{l}\text { Guarro et al., } \\
2003 \text { [7] }\end{array}$ & P. rubrigenum & $55 / \mathrm{M}$ & Renal transplant & $\begin{array}{l}\text { Multiple nodules of } \\
\text { ankle and foot }\end{array}$ & $\begin{array}{l}\text { Excision } \\
\text { Itraconazole } \\
\text { Terbinafine } \\
\text { Fluconazole }\end{array}$ & Not resolved \\
\hline 6 & $\begin{array}{l}\text { Llinas et al., } \\
2005[8]\end{array}$ & $\begin{array}{l}\text { Phaeoacremonium } \\
\text { species }\end{array}$ & $54 / \mathrm{F}$ & $\begin{array}{l}\text { Myelodysplastic } \\
\text { syndrome, IgA } \\
\text { deficiency }\end{array}$ & Olecranon bursitis & $\begin{array}{l}\text { Excision } \\
\text { Itraconazole }\end{array}$ & Resolved \\
\hline 7 & $\begin{array}{l}\text { Baddley et al., } \\
\text { 2006.[9] }\end{array}$ & P.parasiticum & $40 / \mathrm{M}$ & Cardiac transplant & Multiple skin lesions & $\begin{array}{l}\text { Amphotericin B, } \\
\text { Itraconazole } \\
\text { Debridement }\end{array}$ & Died \\
\hline 8 & $\begin{array}{l}\text { Hemashettar et } \\
\text { al., } 2006 \text { [10] }\end{array}$ & P. krajdenii & $41 / \mathrm{M}$ & None & Mycetoma & $\begin{array}{l}\text { Itraconazole } \\
\text { Debridement }\end{array}$ & Recurred \\
\hline 9 & $\begin{array}{l}\text { Marques et al., } \\
2006 \text { [11] }\end{array}$ & P.parasiticum & $49 / \mathrm{M}$ & Renal transplant & $\begin{array}{l}\text { Draining cystic tumors } \\
\text { on the foot }\end{array}$ & $\begin{array}{l}\text { Itraconazole } \\
\text { Amphotericin B }\end{array}$ & Improved \\
\hline 10 & $\begin{array}{l}\text { Huynh et al., } \\
2007 \text { [12] }\end{array}$ & P.parasiticum & $19 / \mathrm{M}$ & $\begin{array}{l}\text { Penetrating globe } \\
\text { injury }\end{array}$ & Endophthalmitis & $\begin{array}{l}\text { Amphotericin B } \\
\text { Voriconazole }\end{array}$ & Improved \\
\hline 11 & $\begin{array}{l}\text { Farina et al., } \\
2007[13]\end{array}$ & P.parasiticum & $41 / \mathrm{M}$ & Kidney transplant & $\begin{array}{l}\text { Subcutaneous nodule on } \\
\text { the forefinger }\end{array}$ & Excision & Resolved \\
\hline 12 & $\begin{array}{l}\text { Baradkar et al., } \\
2009[14]\end{array}$ & P. parasiticum & $26 / \mathrm{F}$ & None & $\begin{array}{l}\text { Subcutaneous abscess on } \\
\text { the forearm }\end{array}$ & $\begin{array}{l}\text { Debridement } \\
\text { Amphotericin B } \\
\text { Itraconazole }\end{array}$ & Resolved \\
\hline 13 & $\begin{array}{l}\text { Sun et al., } 2011 \\
{[15]}\end{array}$ & P. parasiticum & $55 / \mathrm{M}$ & None & Onychomycosis & $\begin{array}{l}\text { Diseased nail was } \\
\text { trimmed off } \\
\text { Topical sulconazole }\end{array}$ & Cured \\
\hline 14 & $\begin{array}{l}\text { Aguilar et al., } \\
\text { 2011.[16] }\end{array}$ & P.parasiticum & $52 / \mathrm{F}$ & $\begin{array}{l}\text { Type } 2 \text { diabetes } \\
\text { hypothyroidism. }\end{array}$ & Eumycetoma & $\begin{array}{l}\text { Surgery (multiple } \\
\text { times), } \\
\text { Itraconazole }\end{array}$ & Improved \\
\hline 15 & $\begin{array}{l}\text { Baradkar et al., } \\
2011[17]\end{array}$ & P. infalitipes & $30 / \mathrm{M}$ & None & $\begin{array}{l}\text { Subcutaneous mass of } \\
\text { the foot }\end{array}$ & $\begin{array}{l}\text { Debridement, } \\
\text { Amphotericin B, } \\
\text { Itraconazole }\end{array}$ & Cured \\
\hline 16 & $\begin{array}{l}\text { Choi et al., } 2011 \\
{[18]}\end{array}$ & $\begin{array}{c}\text { Phaeoacremonium } \\
\text { species }\end{array}$ & $54 / \mathrm{M}$ & Renal transplant & $\begin{array}{l}\text { Subcutaneous mass on } \\
\text { the third finger }\end{array}$ & Excision & Resolved \\
\hline 17 & $\begin{array}{l}\text { Mazzurco et al., } \\
2012 \text { [19] }\end{array}$ & $\begin{array}{l}\text { Phaeoacremonium } \\
\text { species }\end{array}$ & $74 / \mathrm{M}$ & $\begin{array}{l}\text { Rheumatoid } \\
\text { arthritis, on } \\
\text { infliximab }\end{array}$ & Nodule on the leg & $\begin{array}{l}\text { Excision } \\
\text { Itraconazole }\end{array}$ & Resolved \\
\hline 18 & $\begin{array}{l}\text { Furudate et al., } \\
2012[20]\end{array}$ & P. rubrigenum & $76 / F$ & $\begin{array}{l}\text { Still's disease, on } \\
\text { prednisolone }\end{array}$ & $\begin{array}{l}\text { Subcutaneous nodules } \\
\text { on the leg }\end{array}$ & $\begin{array}{l}\text { Debridement, } \\
\text { Itraconazole }\end{array}$ & Resolved \\
\hline 19 & $\begin{array}{l}\text { To et al., } 2012 \\
{[21]}\end{array}$ & P.parasiticum & $69 / \mathrm{M}$ & Diabetes mellitus & $\begin{array}{l}\text { Right knee Pain and } \\
\text { swelling }\end{array}$ & $\begin{array}{l}\text { Arthrotomy and } \\
\text { drainage, } \\
\text { Itraconazole, total } \\
\text { knee replacement }\end{array}$ & Improved \\
\hline 20 & $\begin{array}{l}\text { Guarro et al., } \\
2006[22]\end{array}$ & P. venezuelense & $28 / \mathrm{M}$ & $\begin{array}{c}\text { Chronic myeloid } \\
\text { leukemia }\end{array}$ & Subcutaneous mycoses & Surgical excision & Not known \\
\hline
\end{tabular}


TABLE 2: Invasive and disseminated infections due to Phaeoacremonium species.

\begin{tabular}{|c|c|c|c|c|c|c|c|}
\hline Number & Reference & Species & Age/sex & $\begin{array}{l}\text { Underlying } \\
\text { condition }\end{array}$ & Clinical disease & Treatment & Outcome \\
\hline 21 & $\begin{array}{l}\text { Heath et al., } \\
1997 \text { [23] }\end{array}$ & P.parasiticum & $45 / \mathrm{M}$ & Liver transplant & $\begin{array}{l}\text { Infective endocarditis, } \\
\text { fungemia, and skin } \\
\text { lesion }\end{array}$ & $\begin{array}{l}\text { Amphotericin B, } \\
\text { fluconazole }\end{array}$ & Died \\
\hline 22 & $\begin{array}{l}\text { Wang et al., } \\
2005 \text { [24] }\end{array}$ & P. inflatipes & $\begin{array}{l}18- \\
\text { month } \\
\text { boy }\end{array}$ & Aplastic anemia & Fungemia & Amphotericin B & Died \\
\hline 23 & $\begin{array}{l}\text { Baddley et al., } \\
2006 \text { [9] }\end{array}$ & P. parasiticum & $31 / F$ & Aplastic anemia & Fungemia, skin lesions & Amphotericin B & Died \\
\hline 24 & $\begin{array}{l}\text { McNeil et al., } \\
2011[25]\end{array}$ & P.parasiticum & $24 / \mathrm{M}$ & $\begin{array}{c}\text { Chronic } \\
\text { granulomatous } \\
\text { disease, end-stage } \\
\text { kidney disease }\end{array}$ & Brain abscess & $\begin{array}{l}\text { Amphotericin B, } \\
\text { voriconazole, } \\
\text { caspofungin }\end{array}$ & Died \\
\hline 25 & $\begin{array}{l}\text { To et al., } 2012 \\
\text { [21] }\end{array}$ & P.parasiticum & $26 / \mathrm{M}$ & Renal transplant & Cavitary lesion of lung & $\begin{array}{l}\text { Voriconazole } \\
\text { Caspofungin }\end{array}$ & $\begin{array}{l}\text { Responded, } \\
\text { but died }\end{array}$ \\
\hline 26 & $\begin{array}{l}\text { Shah et al., } 2013 \\
\text { [26] }\end{array}$ & P.parasiticum & $74 / \mathrm{M}$ & Lung transplant & $\begin{array}{l}\text { Lung nodules of native } \\
\text { lung }\end{array}$ & $\begin{array}{l}\text { Caspofungin } \\
\text { Voriconazole }\end{array}$ & Improved \\
\hline 27 & $\begin{array}{l}\text { Larbcharoensub } \\
\text { et al., } 2013 \text { [27] }\end{array}$ & $\begin{array}{l}\text { Scedosporium } \\
\text { apiospermum and } \\
\text { P. parasiticum }\end{array}$ & $49 \mathrm{y} / \mathrm{o}$ & Renal transplant & Multiple brain abscesses & Voriconazole & Improved \\
\hline 28 & Present case & $\begin{array}{c}\text { Phaeoacremonium } \\
\text { species }\end{array}$ & $49 / \mathrm{F}$ & Renal transplant & Lung nodules & Posaconazole & Improved \\
\hline
\end{tabular}

Phaeoacremonium species are typically isolated from thorns, wood, and soil. Human infection can be caused by traumatic implantation or occurs in the setting of immunocompromising conditions. Twenty-seven cases of human infections with Phaeoacremonium species have been reported in the literature. In immunocompetent hosts, Phaeoacremonium has been reported to cause subcutaneous phaeohyphomycosis, osteomyelitis, endophthalmitis, and onychomycosis. Successful outcomes have been achieved with debridement and antifungals (Table 1). In immunocompromised patients, Phaeoacremonium causes more severe disease and has been reported to cause endocarditis, brain abscesses, cavitary lung nodules, and disseminated infections. Disseminated infections in severely immunocompromised hosts are associated with poor outcomes and death (Table 2).

The other dematiaceous fungus isolated from this patient was Dactylaria constricta. It grew only from culture of bronchial fluid and not from the biopsy. Mycobacterium avium-intracellulare complex (MAC) also grew only from the culture of bronchial fluid. Given our patient's exposure history (gardening, exposure to sheds and barns) and no growth of these organisms from the biopsy specimen, it is likely that these organisms were merely colonizers of her respiratory tract and not pathogens. Moreover, resolution of her illness without treatment for MAC suggests that it was not a pathogen.

There is no standard antifungal regimen described for Phaeoacremonium in the literature. Posaconazole is the most recently approved triazole with an extended spectrum of activity against a wide variety of fungi. Posaconazole was chosen over other azoles because it is well tolerated and has a favorable side effect profile and a low potential of drug interactions compared to other azoles. Posaconazole inhibits the metabolism of calcineurin inhibitors. Failure to adjust tacrolimus dosing can result in supratherapeutic levels of tacrolimus and harm the kidney [28]. Our patient responded well to the treatment with no relapse of infection during 4 years of follow-up. Her kidney allograft continues to function well, with creatinine levels ranging between 1 and $1.3 \mathrm{mg} / \mathrm{dL}$.

\section{Conflict of Interests}

The authors declare that there is no conflict of interests regarding the publication of this paper.

\section{References}

[1] U. Damm, L. Mostert, P. W. Crous, and P. H. Fourie, "Novel Phaeoacremonium species associated with necrotic wood of Prunus trees," Persoonia, vol. 20, pp. 87-102, 2008.

[2] L. Mostert, J. Z. Groenewald, R. C. Summerbell et al., "Species of Phaeoacremonium associated with infections in humans and environmental reservoirs in infected woody plants," Journal of Clinical Microbiology, vol. 43, no. 4, pp. 1752-1767, 2005.

[3] N. Singh, F. Y. Chang, T. Gayowski, and I. R. Marino, "Infections due to dematiaceous fungi in organ transplant recipients: case report and review," Clinical Infectious Diseases, vol. 24, no. 3, pp. 369-374, 1997.

[4] A. A. Padhye, M. S. Davis, D. Baer, A. Reddick, K. K. Sinha, and J. Ott, "Phaeohyphomycosis caused by Phaeoacremonium inflatipes," Journal of Clinical Microbiology, vol. 36, no. 9, pp. 2763-2765, 1998. 
[5] T. Matsui, K. Nishimoto, S. Udagawa, H. Ishihara, and T. Ono, "Subcutaneous phaeohyphomycosis caused by Phaeoacremonium rubrigenum in an immunosuppressed patient," Nihon Ishinkin Gakkai Zasshi, vol. 40, no. 2, pp. 99-102, 1999.

[6] K. Kitamura, T. Mochizuki, H. Ishizaki, and R. Fukushiro, "Phaeomycotic cyst caused by Phaeoacremonium parasiticum," Nihon Ishinkin Gakkai Zasshi, vol. 41, no. 2, pp. 89-95, 2000.

[7] J. Guarro, S. H. Alves, J. Gené et al., "Two cases of subcutaneous infection due to Phaeoacremonium spp," Journal of Clinical Microbiology, vol. 41, no. 3, pp. 1332-1336, 2003.

[8] L. Llinas, T. P. Olenginski, D. Bush, R. Gotoff, and V. Weber, "Osteomyelitis resulting from chronic filamentous fungus olecranon bursitis," Journal of Clinical Rheumatology, vol. 11, no. 5, pp. 280-282, 2005.

[9] J. W. Baddley, L. Mostert, R. C. Summerbell, and S. A. Moser, "Phaeoacremonium parasiticum infections confirmed by $\beta$ tubulin sequence analysis of case isolates," Journal of Clinical Microbiology, vol. 44, no. 6, pp. 2207-2211, 2006.

[10] B. M. Hemashettar, B. Siddaramappa, B. S. Munjunathaswamy et al., "Phaeoacremonium krajdenii, a cause of white grain eumycetoma," Journal of Clinical Microbiology, vol. 44, no. 12, pp. 4619-4622, 2006.

[11] S. A. Marques, R. M. P. Camargo, R. C. Summerbell et al., "Subcutaneous phaeohyphomycosis caused by Phaeoacremonium parasiticum in a renal transplant patient," Medical Mycology, vol. 44, no. 7, pp. 671-676, 2006.

[12] T. K. Huynh, L. R. Lee, and D. Ellis, "Late-onset post-traumatic Phaeoacremonium parasiticum endophthalmitis," Clinical and Experimental Ophthalmology, vol. 35, no. 4, pp. 366-368, 2007.

[13] C. Farina, E. Gotti, D. Mouniée, P. Boiron, and A. Goglio, "Phaeoacremonium parasiticum subcutaneous infection in a kidney-transplanted patient successfully treated by surgery," Transplant Infectious Disease, vol. 9, no. 3, pp. 253-255, 2007.

[14] V. Baradkar, M. Mathur, and S. Kumar, "Phaeohyphomycosis of subcutaneous tissue caused by Phaeoacremonium parasiticum," Indian Journal of Medical Microbiology, vol. 27, no. 1, pp. 66-69, 2009.

[15] P.-L. Sun and Y.-M. Ju, "Onychomycosis caused by Phaeoacremonium parasiticum: first case report," Mycoses, vol. 54, no. 2, pp. 172-174, 2011.

[16] A. Aguilar-Donis, E. Torres-Guerrero, R. Arenas-Guzmán et al., "Mycetoma caused by Phaeoacremonium parasiticum-a case confirmed with B-tubulin sequence analysis," Mycoses, vol. 54, no. 5, pp. e615-e618, 2011.

[17] V. Baradkar and S. Kumar, "Subcutaneous granulomatous infection caused by Phaeoacremonium infalitipes on foot," Indian Journal of Dermatology, vol. 56, no. 2, pp. 244-245, 2011.

[18] J. Choi, Y. Lee, H.-S. Chung et al., "Subcutaneous phaeohyphomycosis caused by Phaeoacremonium species in a kidney transplant patient: The first case in Korea," Korean Journal of Laboratory Medicine, vol. 31, no. 3, pp. 201-204, 2011.

[19] J. D. Mazzurco, J. Ramirez, and D. P. Fivenson, "Phaeohyphomycosis caused by Phaeoacremonium species in a patient taking infliximab," Journal of the American Academy of Dermatology, vol. 66, no. 2, pp. 333-335, 2012.

[20] S. Furudate, S. Sasai, Y. Numata et al., "Phaeohyphomycosis caused by Phaeoacremonium rubrigenum in an immunosuppressive patient: a case report and review of the literature," Case Reports in Dermatology, vol. 4, no. 2, pp. 119-124, 2012.
[21] K. K. To, S. K. Lau, A. K. Wu et al., "Phaeoacremonium parasiticum invasive infections and airway colonization characterized by agar block smear and ITS and beta-tubulin gene sequencing.", Diagnostic Microbiology and Infectious Disease, vol. 74, no. 2, pp. 190-197, 2012.

[22] J. Guarro, A. M. Silvestre Jr., G. Verkley et al., "Limitations of DNA sequencing for diagnosis of a mixed infection by two fungi, Phaeoacremonium venezuelense and a Plectophomella sp., in a transplant recipient," Journal of Clinical Microbiology, vol. 44, no. 11, pp. 4279-4282, 2006.

[23] C. H. Heath, J. L. Lendrum, B. L. Wetherall, S. L. Wesselingh, and D. L. Gordon, "Phaeoacremonium parasiticum infective endocarditis following liver transplantation," Clinical Infectious Diseases, vol. 25, no. 5, pp. 1251-1252, 1997.

[24] S.-C. Wang, P.-R. Hsueh, S.-J. Liaw et al., "Fatal fungemia due to Phaeoacremonium inflatipes in a child with severe aplastic anemia," Clinical Infectious Diseases, vol. 40, no. 7, pp. 1067$1068,2005$.

[25] C. J. McNeil, R. F. Luo, H. Vogel, N. Banaei, and D. Y. Ho, "Brain abscess caused by Phaeoacremonium parasiticum in an immunocompromised patient," Journal of Clinical Microbiology, vol. 49, no. 3, pp. 1171-1174, 2011.

[26] S. K. Shah, P. Parto, G. A. Lombard et al., "Probable Phaeoacremonium parasiticum as a cause of cavitary native lung nodules after single lung transplantation," Transplant Infectious Disease, vol. 15, no. 1, pp. E9-E13, 2013.

[27] N. Larbcharoensub, P. Chongtrakool, C. Wirojtananugoon et al., "Treatment of a brain abscess caused by Scedosporium apiospermum and Phaeoacremonium parasiticum in a renal transplant recipient," The Southeast Asian Journal of Tropical Medicine and Public Health, vol. 44, no. 3, pp. 484-489, 2013.

[28] A. Sansone-Parsons, G. Krishna, M. Martinho, B. Kantesaria, S. Gelone, and T. G. Mant, "Effect of oral posaconazole on the pharmacokinetics of cyclosporins and tacrolimus," Pharmacotherapy, vol. 27, no. 6, pp. 825-834, 2007. 


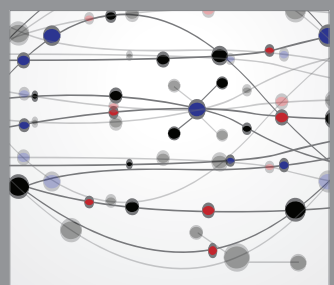

The Scientific World Journal
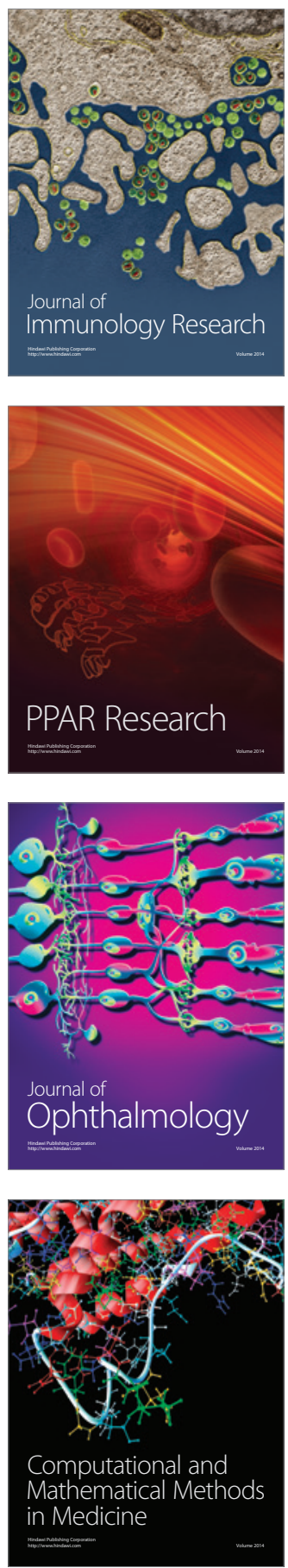

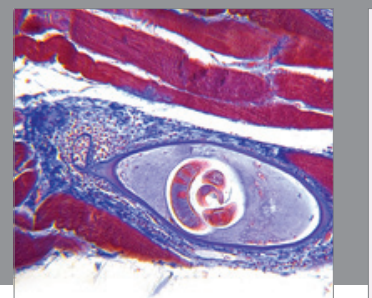

Gastroenterology

Research and Practice
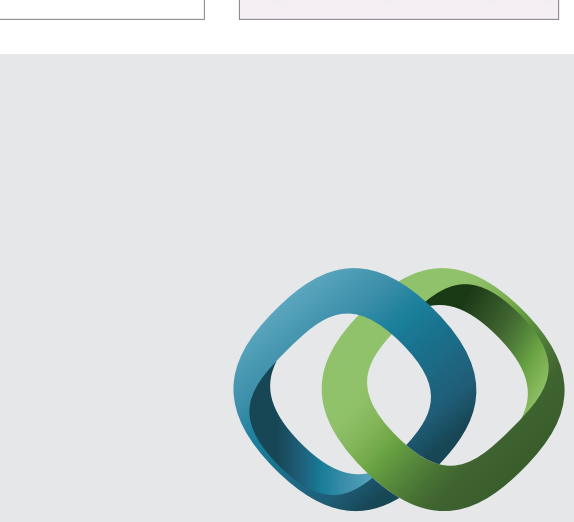

\section{Hindawi}

Submit your manuscripts at

http://www.hindawi.com
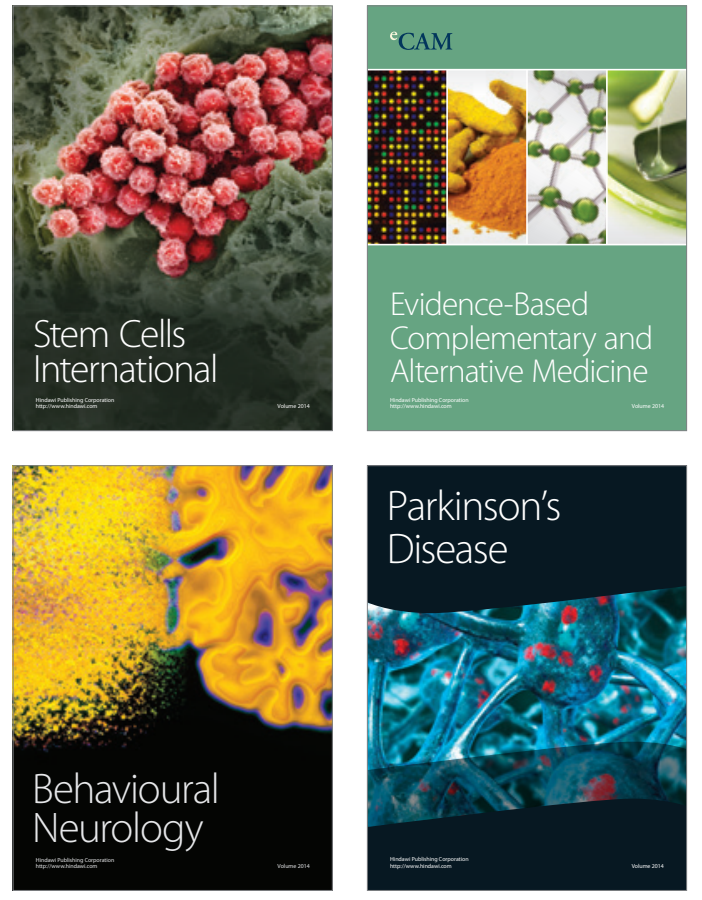
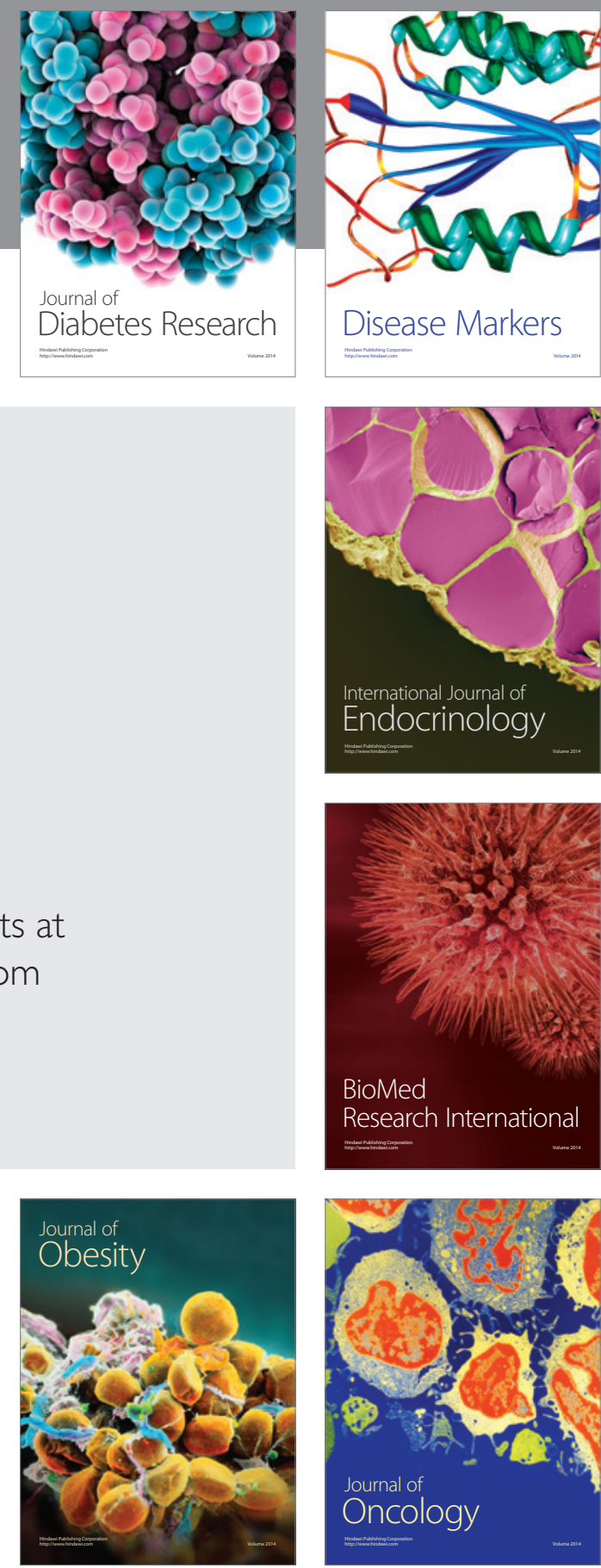

Disease Markers
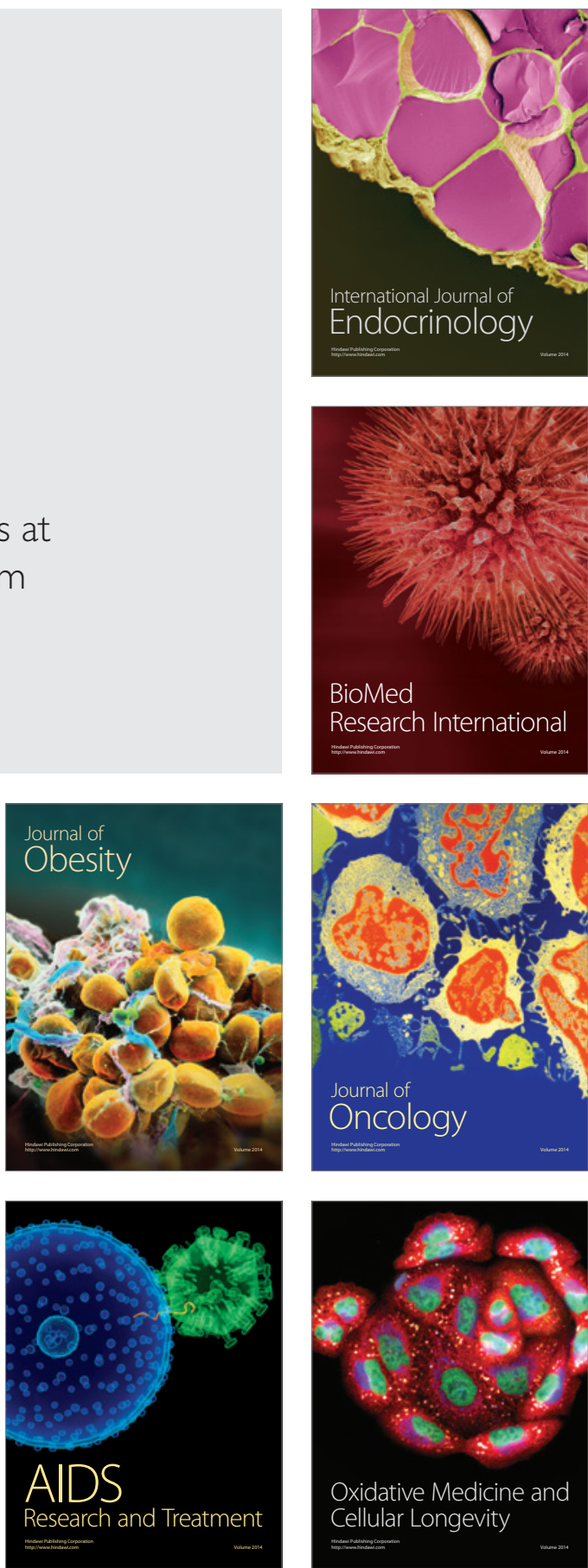